\title{
Le regard de Christine de Pizan sur la jeunesse (à propos du Charles V)
}

Bernard Ribémont

\section{(2) OpenEdition}

1 Journals

Édition électronique

URL : https://journals.openedition.org/crm/904

DOI : $10.4000 / \mathrm{crm} .904$

ISSN : 1955-2424

Éditeur

Honoré Champion

Édition imprimée

Date de publication : 15 février 2000

ISSN : 1272-9752

Référence électronique

Bernard Ribémont, "Le regard de Christine de Pizan sur la jeunesse (à propos du Charles V) ", Cahiers de recherches médiévales [En ligne], 7| 2000, mis en ligne le 03 janvier 2007, consulté le 15 décembre 2022. URL : http://journals.openedition.org/crm/904 ; DOI : https://doi.org/10.4000/crm.904

Ce document a été généré automatiquement le 15 décembre 2022.

Tous droits réservés 


\title{
Le regard de Christine de Pizan sur la jeunesse (à propos du Charles V)
}

\author{
Bernard Ribémont
}

1 Éducatrice, pédagogue, Christine de Pizan prétend l'être à plusieurs reprises dans son œuvre didactique. Soit qu'elle compose un traité d'éducation à part entière, miroir du prince ou de la princesse, comme le Livre du corps de Policie ou le Livre des trois vertus, ou encore qu'elle traite d'histoire, comme dans le Charles $V$, Christine ne perd pas une occasion de donner des enseignements, des exemples. Il est donc logique qu'elle s'adresse, au moins théoriquement, à la jeunesse. De fait, Christine pédagogue expose assez souvent dans son œuvre un certain nombre de considérations qui impliquent un regard sur la jeunesse.

2 C'est ce regard que je me propose d'analyser ici, comme nouveau volet d'une recherche visant à mieux comprendre la psychologie de Christine et l'idéologie qu'elle véhicule ${ }^{1}$. Je m'appuierai pour cela sur un long passage du Livre des fais et bonnes meurs du sage roy Charles $V$ où, à propos de la jeunesse assez déréglée du futur roi (dérèglements sur lesquels elle passe avec pudeur très rapidement $\left.{ }^{2}\right)$, Christine insère une longue digression sur la jeunesse et le passage à l'âge de raison (chapitres IX à XIII).

Le vin nouveau

...et par un gros exemple povons comparer l'omme au vin creu en bonne plante : si advient aucune fois par accident de froidure ou gelee ycellui vin nouvel cueilli estre vert cru et mal proffitable, comme cellui qui n'est mie en boiçon ; mais, lui laissié en tonneaulx croupir au long d'iver, a la gellee, avient souvent que celle verdeur se tourne en bon vin et en meureté couvenable; et semblablement doit retourner homme, aprés toute verdeur de jeunece...(Charles V, I, p. 30).

3 Ce "gros exemple ", comme Christine l'avoue elle-même, sert de conclusion à un assez long exposé du Charles $V$ sur la jeunesse. Parlant du jeune âge du roi, Christine insère en effet dans son texte une longue réflexion théorique sur la jeunesse. C'est de propos délibéré qu'elle se livre à cette digression dont l'introduction est explicite :

Pour ce que le susdit suppost, c'est assavoir, la matiere ou nous sommes entrez du temps de l'aage de jeunece nous donne cause de plus avant dire, sera un petit divulgué, en cestui chapittre, des proprietés d'ycelle, en descripsant, selon les 
autteurs et mon petit engin, ses mouvemens, passions et operacions diverses

(Charles V, I, p. 22)

En s'appuyant sur Aristote et Gilles de Rome, Christine développe donc sa conception des premiers âges de l'homme. Le fait qu'elle consacre cinq chapitres de sa biographie du défunt roi de France à cette question montre bien l'importance que celle-ci revêt à ses yeux.

5 La conclusion du troisième chapitre dévolu à la jeunesse, résumée dans la métaphore $\mathrm{du}$ vin, révèle clairement une conception très traditionnelle, qui oppose jeunesse et vieillesse dans un rapport équivalent à folie et sagesse. Comme le vin nouveau, l'homme jeune est bouillonnant, et son avenir est encore incertain. Il est la proie de ses pulsions immédiates, ce qui pourrait aisément le conduire sur le chemin du vice.

Dans le langage christinien en effet, «jeunesse » est synonyme de dérèglement, dans un sens aussi bien moral que social. Pour Christine, la notion d' «âge de raison » a un sens assez fort, en opposition à la jeunesse où, justement, la raison s'exerce mal : « jeunece ... [est] de soy encline a mains mouvemens hors de raison ». Comme le montre le chapitre qu'elle consacre à la jeunesse du futur sage roi, «jeunesse » est donc une caractéristique humaine à part entière, qui implique un certain nombre de comportements chez l'homme. A tel point que le substantif «jeunesse» apparaît quasiment chez Christine comme une figuration allégorique. Par exemple, la biographe de Charles $\mathrm{V}$ indique que «jeunesse [...] dominoit en lui ».

7 Cette jeunesse est avant tout dangereuse, surtout lorsqu'elle agit sur les jeunes princes. On ne trouvera guère de regard attendri de la mère sur les jeunes humains; Christine est moraliste et éducatrice avant toute chose. Son attention porte donc sur les défauts qu'il s'agit de corriger. En premier lieu, c'est l'orgueil qui guette le jeune prince: «...orgueil, qui leur ramentoit leur haulte poissance, et jeunece, qui les instruit a leur singulier plaisir en tous delis, leur ostent la crainte et regart de toute discipline" (Charles V, I, pp.17-8). On voit ici que la jeunesse apparaît comme un terrain particulièrement favorable au développement des vices, car elle conduit l'homme à céder aisément à son goût pour le plaisir. Ce plaisir est «singulier »; il s'oppose donc à l'intérêt collectif, ce qui est grave pour le prince qui, selon Christine, doit être entièrement préoccupé de ses devoirs envers le royaume. De plus, Christine a tendance à nier toute participation de la vie au principe de plaisir. Lorsqu'il s'agit de prime enfance, c'est avec un certain mépris qu'elle parle des «mignotises maternelles», dont l'enfant princier doit être rapidement tiré, afin d'être séparé de "oisiveté et delices" conçus comme "venin destruiseur de meurs". S'établit ainsi dans la morale christinienne, à propos de jeunesse, un ensemble comportant deux domaines qui s'opposent: d'un côté, la jeunesse et le plaisir, de l'autre la maturité et le travail, l'exercice de la raison. En s'appuyant sur Aristote, Christine va longuement développer cette opposition, en insistant particulièrement sur la sensualité de la jeunesse : celle-ci est le réceptacle de tous les désirs charnels qui l'empêchent de goûter aux «speculatives joyes de l'entendement» (I, p. 23). Ce côté passionnel est essentiellement dû à la domination du corps. La psychologie de la jeunesse s'explique avant tout par une physiologie reposant sur la suprématie du chaud et de l'humide. Comme la plante, la personne jeune est emplie d'une sève qui provoque l'effervescence. C'est pourquoi il est bien souvent difficile de comprendre les attitudes du jeune en proie à de multiples 
mouvements contradictoires. Soumis au corps, celui-ci, rendu aveugle par ses passions, est dissipé : jeunesse s'oppose alors à ordre :

...n'est nulz, qui peust comprendre les divers mouvemens, qui en cellui corps sont compris, lequel, comme passionné d'appetit, sanz ordre, par inclinacion naturele, non cognoiscent encore la lime et correction de raison (...) adonc les volentez agües et sensueles sont comme juge es fais et apetiz alumez et avivez d'yceulz jeunes, qui les rent aveugles et non cognoissans la forme de droit usage (I, p. 24)

Dans ce premier chapitre théorique, Christine développe donc une logique qui aboutit à un jugement moral et social, dans une association, certes traditionnelle, entre corps physique et corps social. Pour cela, elle part d'un constat psychologique et biologique : l'âme est associée au corps, dans un rapport dévalué pour elle. Ce rapport est d'autant plus défavorable que le corps est virulent, ce qui est le cas pendant la jeunesse. Ces débordements du corps agissent ainsi au détriment du jugement de l'âme, ce qui implique une attitude désordonnée qui, elle, nuit à l'ordre établi. La conséquence est donc la nécessité de corriger, de contraindre la jeunesse.

Ces perturbations de l'âme, Christine va en donner un répertoire, ce qui fait l'objet d'une large part du second chapitre du Charles $V$ réservé à la discussion sur la jeunesse. On peut distinguer deux catégories: la première concerne le caractère, la seconde est relative à des questions plus morales. Il existe une relation de cause à effet entre les deux : chaque défaut de caractère imputable à la jeunesse implique un penchant vers le péché ou vers un comportement social indigne ou dangereux. En premier lieu, la jeunesse est impatiente, rétive aux sages conseils, attirée par la satisfaction immédiate des plaisirs et, en conséquence, par l'oisiveté. Elle tombe alors souvent dans ce que Christine nomme elle-même les «foles amours ». De même, la fougue de la jeunesse la conduit à une violence désavouable : la «chaleur du sang» l'entraîne volontiers dans «batailles et riotes ». Toujours dans le registre de l'excès, les jeunes ont tendance à trop parler. Ils ont un sens de l'honneur excessif et mal orienté qui les entraînent à commettre de mauvaises actions : ils ont en effet tendance à confondre «honneur » et "oultrage». La fragilité de leur jugement les conduit aussi à aimer leurs «mortelz anemis » et à écouter avec attention les «aduleurs ou flateurs». Le jeune est alors soumis à de «mauvais consaulz ».

10 Ce qui frappe le plus dans le discours christinien est donc l'opposition de la jeunesse à la raison: jeunesse est pour elle le temps de folie. Chez Christine, même si une telle dimension existe, il ne s'agit pas non plus de simple métaphore, s'appuyant sur un lieu commun du langage. On ne peut en effet qu'être frappé par les nombreuses occurrences du termes «folie » et de ses dérivés et synonymes. Et l'introduction «aristotélicienne » du discours christinien éclaire bien une démarche que l'intégralité du raisonnement et de l'exemplaire confirme : «folie » est à comprendre avant tout dans le sens médical du terme. C'est pourquoi, Christine emprunte au vocabulaire des théories humorales. Ces humeurs abondent, désordonnées dans le corps jeune, et agissent par contrecoup sur l'âme et le comportement. Dépourvu d'expérience, le jeune ne peut maîtriser ses passions et il est effectivement fou, dans la mesure où il agit largement selon le dérèglement des humeurs :

Infinis mouvemens habondent es cuers des jeunes sanz frain de raison, qui est le regart de la fin de toutes choses, es uns plus, es aultres moins, selon leur diverses complexions, lesquelles causent es aucuns joyes, es autres riotes et melancolie, si comme aux sanguins soulas et esbatemens, et aux melancoliques ou coleriques riotes et despiz, et partout y a infinis perilz (I, p. 26). 
11 Ce passage, compris entre deux «infinis", est particulièrement révélateur de la démarche de Christine de Pizan. Partant d'un constat physiologique, qui permet l'utilisation d'un vocabulaire à caractère scientifique, Christine aboutit à des considérations morales sur les dangers qui guettent la jeunesse, partie que la pédagogue qu'elle est toujours entend bien souligner. On retrouve ici donc des constantes du discours didactique christinien : tout d'abord, une analyse des causes, dans laquelle un référent scientifique et encyclopédique est volontiers convoqué, qui participe aussi de l'affirmation de l'autorité de l'écrivain. Cette analyse, qui repose aussi sur la référence à des autorités anciennes, est suivie de considérations morales et didactiques, débouchant sur une série de conseils, pour lesquels Christine s'appuie aussi sur les exempla fournis par les autorités. C'est cette seconde partie du discours qui est particulièrement développée, Christine entendant surtout éduquer d'une part et, d'autre part, en ce qui concerne le Charles $V$ plus précisément, montrer comment le jeune roi avait eu la capacité rapide de prendre le chemin de la raison.

Le parcours du jeune roi vers la sagesse est donc une lutte contre la domination de "jeunesse", lutte qui correspond au cheminement vers la maturité : le vin nouveau ${ }^{3}$ devra perdre sa jeunesse et ses turbulences.

La force de l'éducation

domination de "jeunesse" sur le prince est, selon Christine, le fruit d'une mauvaise éducation, car le jeune Charles était entouré de mauvais administrateurs. On voit ici la réunion, possible autour de la personne du jeune roi, du topos des mauvais conseillers et administrateurs, si fréquent dans le discours politique médiéval, et de celui des mauvais précepteurs responsables de la corruption du prince. Notons cependant qu'à propos du jeune roi, Christine ne peut être complètement affirmative : elle «suppose» que seule la présence de mauvais éducateurs dans l'entourage du jeune prince peut expliquer la mauvaise conduite de celui-ci. C'est l'occasion pour la pédagogue de réaffirmer l'importance première de l'éducation, tout particulièrement chez les puissants. Ces derniers en effet doivent toujours montrer l'exemple et ils doivent donc être particulièrement bien éduqués dans leur jeune âge « ...tout aussi que seigneurie humaine est rigle des autres estas, est raison qu'elle soit regulee et remplie des precieux joyaulx de vertus et de l'entendement, et pour ce les parens, obviens a telz inconveniens, doivent plus singulierement procurer a leurs enfens bonne compaignie, sage et honneste, et prendre garde a la discipline des meurs» (I, p. 18). C'est une constante dans l'enseignement de Christine, que l'on retrouve dans les Trois vertus ou dans Policie, que ces conseils à veiller minutieusement au choix des précepteurs des enfants princiers ${ }^{4}$.

On l'a vu, Christine oppose nettement l'univers des plaisirs immédiats procurés par la satisfaction des sens aux «speculatives joyes de l'entendement». L'homme ne peut accéder à ces plaisirs-là que par les vertus de l'enseignement ; c'est pourquoi Christine insiste fortement sur la nécessité absolue d'une éducation solide. Celle-ci doit être sévère - ou, plus précisément, ferme -, car, initialement, elle contrarie les penchants naturels de la jeunesse pour l'oisiveté et la facilité : «O Dieux ! Comment voions nous les jeunes gens adouler et entrister quant correction, quoyqu'elle leur soit salutaire, leur est presentee, laquelle reçoivent comme opprobre et chose injurieuse " (I, pp. 24-5). Cependant, autre constante du discours christinien, c'est surtout par l'exemple que le précepteur doit éduquer. Dans ce passage théorique du Charles V, Christine énonce des 
idées rigoureusement analogues à celles de Policie ou des Trois vertus: mieux vaut exhorter à la vertu que battre: les éducateurs «doivent plus corrigier par bons exemples et paroles introduisans a bonne meurs que par verberacions ou bateures maistriseuses ». Il y a donc une réelle adéquation entre la pédagogie prônée par Christine et son discours didactique: Christine croit fermement à la valeur de l'exemple. Et, comme le révèle particulièrement Policie, éduquer, c'est fournir le plus d'exemples édifiants possibles. Si son œuvre est dense en exemples, ce n'est donc pas uniquement par souci, bien réel cependant, d'affirmer son autorité ; c'est aussi que Christine conçoit la parole exemplaire comme le meilleur soutien de l'enseignement et de la correction des mœurs. C'est pourquoi sa parole "politique», son perpétuel discours en faveur de la paix, qu'elle considère toujours selon une orientation morale, s'appuient systématiquement sur l'exemple.

Il y a donc une relative douceur dans la conception christinienne de l'éducation du jeune prince. De façon plus générale - et l'on retrouve ici encore un élément du discours politique et moral-, Christine fait preuve d'un certain optimisme, tant sa croyance au pouvoir de l'éducation est forte. Certes, la jeunesse est un âge ingrat, qu'il faut systématiquement corriger. Mais il est du devoir des adultes de ne rien négliger en ce domaine et de ne pas céder à une facilité qui consiste à penser, devant certains agissements des jeunes, que tout est perdu:

Ainsi ne doit homme nullement jugier, tant voie le jeune foloier ou desvoyé en quelconque voie dissolue, que jamais bien ne fera et que estre deust chaciez comme publiquan, mais doit dire d'yceulz, si comme il est recité es paroles de Jhesu-Crist : «Pere, pardonnez leur, car ilz ne scevent qu'ilz font!», et rappeller les doit on par moderacions propices (I, p. 29)

D'ailleurs, Christine termine la partie qu'elle consacre à la jeunesse proprement dite par quelques exempla.

La conception que Christine a de la jeunesse est donc traditionnelle. Elle n'a pas de regard appuyé sur cette période de la vie, considérée, toujours traditionnellement, comme un âge ne pouvant rien produire de bon, mais seulement capable de recevoir ce que les adultes, hommes de raison, peuvent lui apporter. Comme nous l'avons remarqué ailleurs ${ }^{5}$, la mère qu'est Christine dans la réalité n'apparaît guère chez l'écrivain, entièrement masquée par l'éducatrice. Observer la jeunesse, c'est donc l'éduquer. Attitude essentielle, surtout en ce qui concerne les enfants princiers auxquels Christine s'intéresse particulièrement-, car ces derniers sont appelés à occuper des places importantes dans la société et, à ce titre, ils doivent servir d'exemple. A tout niveau de la pensée, comme de la méthode didactique christiniennes, on retrouve la valeur de l'exemple. C'est pourquoi l'éducation qu'elle propose s'appuie toujours sur deux pôles : la vertu des éducateurs (ils doivent être exemplaires!) et la parole exemplaire. Partant $d u$ "vin nouveau ", d'une jeunesse soumise aux passions $d u$ corps - ces termes compris dans une acception médicale-, Christine propose un parcours vers l'âge adulte qu'elle conçoit comme un apprentissage de l'usage de la raison. C'est grâce à l'exemple, aussi bien celui fourni par la vie des éducateurs (exemple moral) que celui indiqué par les autorités (exemple culturel et moral) que le jeune se détournera des plaisirs immédiats pour les «greigneurs estudes» qui, seules, peuvent procurer une satisfaction authentique et durable. Ainsi le regard de Christine sur la jeunesse correspond bien aux conceptions générales de son discours didactique, qui, somme toute, se révèle toujours optimiste, parce que reposant sur le ferme espoir 
en une toujours possible amélioration morale produite par une éducation que, en tous sens, Christine veut exemplaire.

\section{NOTES}

1. Cet article fait ainsi suite à deux autres contributions : «L'image de la mère chez Christine de Pizan », à paraître dans les Mélanges A.J. Kennedy ; et «Christine et la nouveauté ", à paraître dans les Mélanges L. Dulac.

2. «...n'est a mon propos et ne quier faire grant narracion sur les fais de l'adolescence du dit roy ".

3. Cette métaphore du vin qui conclut la partie où Christine se livre à une analyse de la jeunesse prend toute son importance par rapport à la construction «scientifique» du discours christinien. En effet, le vin, liquide à l'alchimie complexe, peut être aussi vu, dans ce cadre, comme une métaphore des humeurs du corps humain.

4. Voir notre article "Jeanne de Schomberg, lectrice de Christine de Pizan? ", à paraître dans les Mélanges Eric Hicks.

5. Voir «Christine et l'image de la mère », à paraître dans les Mélanges A. Kennedy.

\section{AUTEUR}

\section{BERNARD RIBÉMONT}

C.E.M.O. - Université d'Orléans 